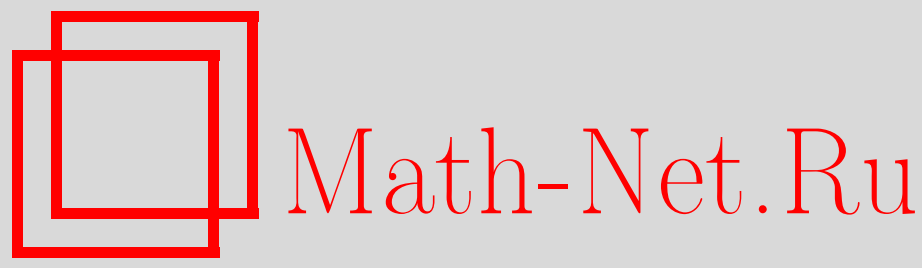

Н. М. Плакида, Двухвременные функции Грина и диаграммная техника, ТМФ, 2011, том 168, номер 3, 518-535

DOI: https://doi.org/10.4213/tmf6698

Использование Общероссийского математического портала Math-Net.Ru подразумевает, что вы прочитали и согласны с пользовательским соглашением http: //www.mathnet.ru/rus/agreement

Параметры загрузки:

IP: 54.147 .182 .235

26 апреля 2023 г., $17: 25: 29$

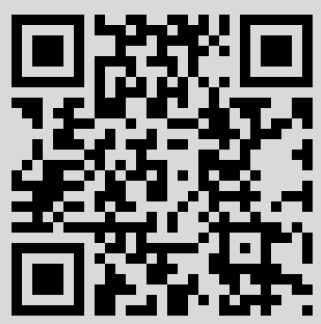




\title{
ФИЗИКА
}

Том 168, № 3

сентябрь, 2011

2011 г.

Н. М. Плакида*

\section{ДВУХВРЕМЕННЫЕ ФУНКЦИИ ГРИНА И ДИАГРАММНАЯ ТЕХНИКА}

\begin{abstract}
Обсуждаются вычисления динамической спиновой восприимчивости и корреляционных функций в модели Гейзенберга, основанные на составлении уравнений движения для двухвременных функций Грина. На основе метода проектирования типа Мори выводится точное уравнение Дайсона с массовым оператором в виде многочастичных функций Грина. Вычисление массового оператора в приближении взаимодействующих мод в ферромагнитной фазе приводит к результатам температурной диаграммной техники, в том числе дает корректную формулу для низкотемпературной намагниченности. Рассмотрено также вычисление спектра спиновых флуктуаций в парамагнитной фазе в рамках метода уравнений движения для функции релаксации.
\end{abstract}

Ключевые слова: динамическая спиновая восприимчивость, модель Гейзенберга, запаздывающие и опережающие функции Грина, расцепление Тябликова, диаграммная техника для спиновых функций Грина.

\section{1. ВВЕДЕНИЕ}

Двухвременные температурные функции Грина (ФГ) (запаздывающие и опережающие), введенные в статистическую физику Боголюбовым и Тябликовым [1], [2], оказались весьма эффективным методом изучения спектра возбуждений и фазовых переходов в многочастичных системах [3]. Применение этого метода в спиновых системах позволило Тябликову уже в простейшем приближении среднего поля построить интерполяционную теорию для описания намагниченности ферромагнетика в широком интервале температур, включая парамагнитную фазу [2], [4]. Некоторым недостатком развитой теории было появление в формуле для низкотемпературной намагниченности вкладов порядка $T^{3}$, обусловленных "кинематическим взаимодействием", что противоречит теории Дайсона [5] и результатам спиновой диграммной техники, разработанной позднее [6]-[9] (см. также монографии [10]-[12]).

В дальнейшем было показано, что в рамках более строгой теории [13], учитывающей затухание спиновых волн, удается получить корректную формулу для низкотемпературной намагниченности [14], [15]. На основе метода проектирования типа Мори [16] при дифференцировании ФГ по двум временам было выведено точное

* Объединенный институт ядерных исследований, Дубна, Московская обл., Россия. E-mail: plakida@theor.jinr.ru 
уравнение Дайсона [17]. Приближенное вычисление массового оператора в этом уравнении позволяет воспроизвести результаты диаграммной техники [7] и получить корректную формулу для низкотемпературной намагниченности [18], как и в работах [14], [15]. Общий метод получения формально точной системы уравнений для двухвременных ФГ при дифференцировании ФГ по двум временам был предложен в работе [19] и затем использован для построения теории спиновых флуктуаций в парамагнитной фазе [20]. Вычисление массового оператора ФГ в приближении взаимодействующих мод позволило получить интерполяционную формулу для описания спектра спиновых возбуждений в ферромагнитной и парамагнитной областях [21].

На основе метода двухвременных ФГ была разработана также общая теория динамической спиновой восприимчивости в антиферромагнетике при наличии дырочных возбуждений в рамках $t-J$ модели [22]. Вычисление спектра спиновых флуктуаций на основе развитой теории было проведено как в нормальной фазе [23], так и в сверхпроводящей фазе [24] при учете вкладов более высокого порядка в массовом операторе, отсутствующих в температурной диаграммной технике [25]. Учет этих членов необходим для построения корректной теории магнитной резонансной моды, наблюдаемой в высокотемпературных купратных сверхпроводниках (см. монографию [26]).

В настоящей работе мы обсуждаем общий метод вычисления динамической спиновой восприимчивости на примере модели Гейзенберга, используя метод проектирования типа Мори в уравнениях для ФГ и приближение взаимодействующих мод для вычисления массового оператора. В разделе 2 обсуждается общая теория, которая используется для вычисления спектра спиновых волн и намагниченности в ферромагнитной фазе. Спектр спиновых флуктуаций в парамагнитной фазе для антиферромагнитной модели Гейзенберга рассмотрен в разделе 3 .

\section{2. ФЕРРОМАГНИТНАЯ МОДЕЛЬ ГЕЙЗЕНБЕРГА}

2.1. Уравнение Дайсона. В качестве примера применения техники уравнений движения для двухвременных ФГ рассмотрим изотропную ферромагнитную модель Гейзенберга для спиновых операторов $S=1 / 2$ :

$$
H=-h \sum_{i} S_{i}^{z}-\frac{1}{2} \sum_{i \neq j} J_{i j}\left(\mathbf{S}_{i} \mathbf{S}_{j}\right)
$$

где $h=\mu_{\mathrm{B}} H^{z}$ - зеемановская энергия спина во внешнем магнитном поле $H^{z}$ и $J_{i j}-$ обменное взаимодействие для спинов на узлах решетки $i \neq j$. Спектр спиновых возбуждений определяется динамической восприимчивостью для поперечных компонент оператора спина $S_{i}^{ \pm}=S_{i}^{x} \pm i S_{i}^{y}$ :

$$
\chi(\mathbf{q}, \omega)=-\left\langle\left\langle S_{\mathbf{q}}^{+} \mid S_{\mathbf{q}}^{-}\right\rangle_{\omega}=-\frac{1}{N} \sum_{i, j} e^{-i \mathbf{q}(\mathbf{i}-\mathbf{j})}\left\langle\left\langle S_{i}^{+} \mid S_{j}^{-}\right\rangle_{\omega} .\right.\right.
$$

Здесь мы ввели фурье-образ запаздывающей двухвременной коммутаторной ФГ в обозначениях Зубарева [3]:

$$
\langle\langle A \mid B\rangle\rangle_{\omega}=-i \int_{0}^{\infty} d t e^{i \omega t}\langle[A(t), B]\rangle, \quad \operatorname{Im} \omega>0,
$$


где $A(t)=e^{i H t} A e^{-i H t}$ и $\langle A B\rangle$ - статистическое среднее от оператора $A B$ при температуре $T$. В дальнейшем мы пользуемся системой единиц, в которой $\hbar=1, k_{\mathrm{B}}=1$ и $\beta=1 / k_{\mathrm{B}} T=1 / T$.

Для вычисления двухвременной спиновой $Ф Г$ в (2) воспользуемся уравнением движения

$$
\omega\left\langle\left\langle S_{i}^{+} \mid S_{j}^{-}\right\rangle_{\omega}=2\left\langle S_{i}^{z}\right\rangle \delta_{i j}+\left\langle\left\langle i \dot{S}_{i}^{+} \mid S_{j}^{-}\right\rangle\right\rangle_{\omega}\right.
$$

где для операторов спина $i \dot{S}_{i}^{ \pm}=i(d / d t) S_{i}^{ \pm}$имеем уравнение

$$
\pm i \dot{S}_{i}^{ \pm}=\left[S_{i}^{ \pm}, H\right]=h S_{i}^{ \pm}+\sum_{m} J_{i m}\left(S_{m}^{z} S_{i}^{ \pm}-S_{i}^{z} S_{m}^{ \pm}\right)
$$

В правой части этого уравнения появляются многочастичные спиновые операторы, в которых удобно провести разделение когерентных и некогерентных процессов в распространении спиновых возбуждений с помощью некоторой проекционной техники. При этом устраняется произвол в расцеплении $Ф Г$, возникающий в обычном методе обрыва цепочки уравнений для двухвременных ФГ [3]. Для исключения секулярных членов из временны́х корреляционных функций наиболее часто используется метод Мори [16], основанный на введении неприводимых операторов, и метод неприводимого оператора Лиувилля (см., например, работу [27]). В то время как вычисление неприводимых (irreducible) операторов является достаточно простым, вычисление временно́й зависимости с помощью неприводимого оператора Лиувилля представляет определенные трудности и иногда приводит к необоснованным результатам (см. работу [28]). Церковников предложил общий метод проектирования для временнь́х $Ф Г$ [19], в котором в качестве скалярного произведения используется временна́я ФГ. При таком подходе спроектированные ФГ удовлетворяют формально точной цепочке уравнений, обрыв которой на некотором шаге позволяет получить приближенное выражение для исходной $Ф Г$.

Здесь мы применим обычную версию проекционной техники типа Мори для ФГ [29]. Применение этого метода позволяет достаточно просто получить уравнение Дайсона в модели Гейзенберга [17]. Метод основан на введении неприводимых операторов со скалярным произведением, определенным для статических корреляционных функций. Для этого выделим в уравнении движения (5) линейную часть и ортогональное к ней дополнение $Z_{i}^{+(\mathrm{irr})}$ согласно правилу проектирования:

$$
\left[S_{i}^{+}, H\right]=h S_{i}^{+}+\sum_{l} E_{i l} S_{l}^{+}+Z_{i}^{+(\mathrm{irr})}, \quad\left\langle\left[Z_{i}^{+(\mathrm{irr})}, S_{j}^{-}\right]\right\rangle=0
$$

Это уравнение определяет матрицу частот $E_{i j}$ и соответствующую нулевую ФГ в обобщенном приближении среднего поля (ПСП) [17] (см. также работу [14]):

$$
\begin{gathered}
E_{i j}=\frac{\left\langle\left[\left[S_{i}^{+}, H\right], S_{j}^{-}\right]\right\rangle}{\left\langle\left[S_{i}^{+}, S_{i}^{-}\right]\right\rangle}=\sum_{m} J_{i m}\left[\delta_{i, j}-\delta_{j, m}\right] \frac{2\left\langle S_{i}^{z} S_{m}^{z}\right\rangle+\left\langle S_{i}^{-} S_{m}^{+}\right\rangle}{2\left\langle S_{i}^{z}\right\rangle} \\
G_{i j}^{(0)}(\omega)=\left\langle\left\langle S_{i}^{+} \mid S_{j}^{-}\right\rangle\right\rangle_{\omega}^{(0)}=2\left\langle S_{i}^{z}\right\rangle\left\{(\omega-h) \delta_{i j}-E_{i j}\right\}^{-1}
\end{gathered}
$$


Вводя далее фурье-разложение по волновым векторам q в пространстве обратной решетки согласно (2), получим

$$
\begin{gathered}
G^{(0)}(\mathbf{q}, \omega)=\left\langle\left\langle S_{\mathbf{q}}^{+} \mid S_{-\mathbf{q}}^{-}\right\rangle_{\omega}^{(0)}=\frac{\sigma}{\omega-h-E_{\mathbf{q}}}\right. \\
E_{\mathbf{q}}=\left\langle S_{i}^{z}\right\rangle\left(J_{0}-J_{\mathbf{q}}\right)+\frac{1}{\sigma N} \sum_{\mathbf{k}}\left(J_{\mathbf{k}}-J_{\mathbf{k}-\mathbf{q}}\right)\left[2 K^{z z}(\mathbf{k})+K^{-+}(\mathbf{k})\right] .
\end{gathered}
$$

Здесь введены средняя намагниченность системы $\sigma=\left\langle S_{i}^{z}\right\rangle / S=2\left\langle S_{i}^{z}\right\rangle$, фурье-компонента обменного взаимодействия $J_{\mathbf{q}}=(1 / N) \sum_{i, j} e^{-i \mathbf{q}(\mathbf{i}-\mathbf{j})} J_{i, j}$ и корреляционные функции флуктуаций продольных компонент спина $\delta S_{i}^{z}=S_{i}^{z}-\left\langle S_{i}^{z}\right\rangle$,

$$
\left\langle\delta S_{i}^{z} \delta S_{j}^{z}\right\rangle=\frac{1}{N} \sum_{\mathbf{q}} e^{i \mathbf{k}(\mathbf{i}-\mathbf{j})} K^{z z}(\mathbf{k}),
$$

и поперечных компонент спина,

$$
\left\langle S_{i}^{-} S_{j}^{+}\right\rangle=\frac{1}{N} \sum_{\mathbf{k}} e^{\mathbf{k}(\mathbf{i}-\mathbf{j})} K^{-+}(\mathbf{k}) .
$$

Первый член в формуле (10) соответствует ПСП Тябликова [1], [4], в котором не учитываются вклады от статических флуктуаций спинов. Такие же результаты для спектра спиновых возбуждений в ПСП (обобщенном приближении Хартри-Фока) были получены в работе [14] (более подробно см. дополнение в книге [2]).

Учитывая представление (6), запишем уравнение движения (4) для ФГ в виде

$$
\sum_{m}\left[(\omega-h) \delta_{i, m}-E_{i m}\right]\left\langle\left\langle S_{m}^{+} \mid S_{j}^{-}\right\rangle\right\rangle_{\omega}=\sigma \delta_{i j}+\left\langle\left\langle Z_{i}^{+(\mathrm{irr})} \mid S_{j}^{-}\right\rangle\right\rangle_{\omega} .
$$

Вычислим многочастичную ФГ в правой части этого уравнения, дифференцируя ее по второму времени $t^{\prime}$, т. е. вычисляя $-i\left(d / d t^{\prime}\right)\left\langle\left\langle Z_{i}^{+(\mathrm{irr})}(t) \mid S_{j}^{-}\left(t^{\prime}\right)\right\rangle\right\rangle$. Для фурье-компоненты ФГ получим уравнение

$$
\begin{aligned}
\omega\left\langle\left\langle Z_{i}^{+(\mathrm{irr})} \mid S_{j}^{-}\right\rangle\right\rangle_{\omega}= & \left\langle\left\langle Z_{i}^{+(\mathrm{irr})} \mid-i \dot{S}_{j}^{-}\right\rangle\right\rangle_{\omega}=h\left\langle\left\langle Z_{i}^{+(\mathrm{irr})} \mid S_{j}^{-}\right\rangle\right\rangle_{\omega}+ \\
& +\sum_{m} E_{j m}\left\langle\left\langle Z_{i}^{+(\mathrm{irr})} \mid S_{m}^{-}\right\rangle\right\rangle_{\omega}+\left\langle\left\langle Z_{i}^{+(\mathrm{irr})} \mid Z_{j}^{-(\mathrm{irr})}\right\rangle\right\rangle_{\omega},
\end{aligned}
$$

где мы учли, что $\left\langle\left[Z_{i}^{+(\mathrm{irr})}, S_{j}^{-}\right]\right\rangle=0$, и ввели неприводимую часть оператора $-i \dot{S}_{j}^{-}$ аналогично уравнению (6). Учитывая определение нулевой ФГ (8), (9), мы можем представить решение системы уравнений (11), (12) для фурье-компоненты ФГ $G(\mathbf{q}, \omega)=\left\langle\left\langle S_{\mathbf{q}}^{+} \mid S_{\mathbf{q}}^{-}\right\rangle_{\omega}\right.$ в виде

$$
G(\mathbf{q}, \omega)=G^{(0)}(\mathbf{q}, \omega)+G^{(0)}(\mathbf{q}, \omega) T(\mathbf{q}, \omega) G^{(0)}(\mathbf{q}, \omega),
$$

где введена матрица рассеяния

$$
T(\mathbf{q}, \omega)=\frac{1}{\sigma}\left\langle\left\langle Z_{\mathbf{q}}^{+(\mathrm{irr})} \mid Z_{\mathbf{q}}^{-(\mathrm{irr})}\right\rangle\right\rangle_{\omega} \frac{1}{\sigma} .
$$


Для устранения секулярных членов во временно́й зависимости в матрице рассеяния определим собственную часть (proper part) этой матрицы $T^{(\mathrm{pp})}(\mathbf{q}, \omega)=\Sigma(\mathbf{q}, \omega)$ с помощью уравнения

$$
T(\mathbf{q}, \omega)=\Sigma(\mathbf{q}, \omega)+\Sigma(\mathbf{q}, \omega) G^{(0)}(\mathbf{q}, \omega) T(\mathbf{q}, \omega) .
$$

Как и в методе Мори, это уравнение исключает вклад от свободного распростране-

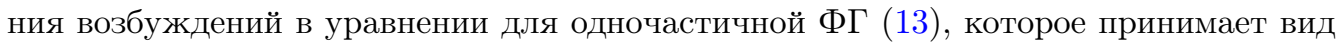
уравнения Дайсона для полной $Ф Г$,

$$
G(\mathbf{q}, \omega)=G^{(0)}(\mathbf{q}, \omega)+G^{(0)}(\mathbf{q}, \omega) \Sigma(\mathbf{q}, \omega) G(\mathbf{q}, \omega)=\frac{\sigma}{\omega-h-E_{\mathbf{q}}-M(\mathbf{q}, \omega)},
$$

с массовым оператором

$$
M(\mathbf{q}, \omega)=\sigma \Sigma(\mathbf{q}, \omega)=\left\langle\left\langle Z_{\mathbf{q}}^{+(\mathrm{irr})} \mid Z_{\mathbf{q}}^{-(\mathrm{irr})}\right\rangle\right\rangle_{\omega}^{(\mathrm{pp})} \frac{1}{\sigma} .
$$

Спектр спиновых возбуждений определяется мнимой частью восприимчивости (2), или спектральной плотностью ФГ (14):

$$
A(\mathbf{q}, \omega)=-\frac{1}{\sigma \pi} \operatorname{Im} G(\mathbf{q}, \omega+i \delta)=-\frac{1}{\pi} \frac{M^{\prime \prime}(\mathbf{q}, \omega)}{\left[\omega-h-E_{\mathbf{q}}-M^{\prime}(\mathbf{q}, \omega)\right]^{2}+\left[M^{\prime \prime}(\mathbf{q}, \omega)\right]^{2}},
$$

где $M(\mathbf{q}, \omega+i \delta)=M^{\prime}(\mathbf{q}, \omega)+i M^{\prime \prime}(\mathbf{q}, \omega)$. Здесь $M^{\prime}(\mathbf{q}, \omega)$ - реальная часть и $M^{\prime \prime}(\mathbf{q}, \omega)$ мнимая часть массового оператора. Когерентное распространение спиновых возбуждений описывается спектром возбуждений в обобщенном ПСП $E_{\mathbf{q}}$, в то время как массовый оператор $M(\mathbf{q}, \omega)$ определяет некогерентную часть спектра, обусловленную неупругими процессами рассеяния. Последние определяют затухание спиновых возбуждений: $\Gamma(\mathbf{q}, \omega)=-M^{\prime \prime}(\mathbf{q}, \omega)>0$.

В спиновой диаграммной технике для ФГ используется представление Ларкина с эффективным взаимодействием [6]-[8], [10]. В методе проектирования Мори получается точное уравнение Дайсона (14) с массовым оператором в виде многочастичной

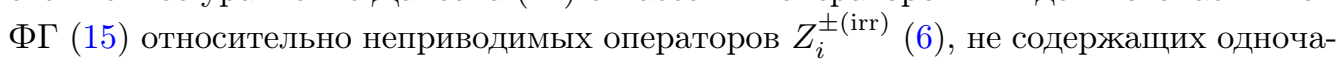
стичных операторов. Оба представления оказываются эквивалентными, если провести точное вычисление всех вкладов [12]. Однако при приближенном вычислении массового оператора и эффективного взаимодействия результаты могут отличаться. Как показано ниже, при учете диаграмм низшего порядка результаты для спектра спиновых волн и корреляционных функций совпадают.

2.2. Приближенное вычисление массового оператора. Для вычисления массового оператора воспользуемся приближением взаимодействующих мод, которое эквивалентно приближению непересекающихся диаграмм. Используя спектральные представления для ФГ [3], выразим массовый оператор (15) через неприводимую часть многочастичной двухвременной корреляционной функции:

$$
\begin{aligned}
M(\mathbf{q}, \omega)= & \frac{1}{2 \pi \sigma} \int_{-\infty}^{\infty} \frac{d \omega^{\prime}}{\omega-\omega^{\prime}}\left(e^{\beta \omega^{\prime}}-1\right) \int_{-\infty}^{\infty} d t e^{-i \omega^{\prime} t} \times \\
& \times \frac{1}{N} \sum_{i, j} e^{-i \mathbf{q}(\mathbf{j}-\mathbf{i})}\left\langle Z_{j}^{-(\mathrm{irr})}(t) \mid Z_{i}^{+(\mathrm{irr})}\right\rangle^{(\mathrm{pp})} .
\end{aligned}
$$


Учитывая определение неприводимой части оператора тока $Z_{i}^{ \pm(\mathrm{irr})}(6)$ в уравнениях движения (5), запишем многочастичную двухвременную функцию в правой части равенства (17) в виде

$$
\begin{aligned}
\left\langle Z_{j}^{-(\mathrm{irr})}(t) \mid Z_{i}^{+(\mathrm{irr})}\right\rangle^{(\mathrm{pp})}= & \sum_{l, m} J_{j m} J_{l i}\left\langle\left[\delta S_{m}^{z}(t) S_{j}^{-}(t)-\delta S_{j}^{z}(t) S_{m}^{-}(t)\right] \times\right. \\
& \left.\times\left[\delta S_{l}^{z} S_{i}^{+}-\delta S_{i}^{z} S_{l}^{+}\right]\right\rangle .
\end{aligned}
$$

В этом выражении вклады вида $\sum_{l} E_{i l} S_{l}^{+}$в формуле для неприводимого оператоpa (6) были исключены в соответствии с определением собственной части многочастичной корреляционной функции, которая не должна содержать одночастичных функций типа $\left\langle S_{i}^{-}(t) S_{j}^{+}\right\rangle$.

В приближении взаимодействующих мод предполагается, что распространение "одетых" спиновых флуктуаций $\delta S_{m}^{z}$ и $S_{j}^{ \pm}$происходит независимо. Это приближение соответствует самосогласованному борновскому приближению, в котором пренебрегают перенормировкой вершин, и эквивалентно вычислению скелетной диаграммы в низшем приближении по взаимодействию. В предположении о независимом распространении спиновых возбуждений многочастичные временные корреляционные функции записываются в виде произведения соответствующих одночастичных функций:

$$
\left\langle\delta S_{\mathbf{k}}^{z}(t) S_{\mathbf{q}}^{-}(t) \delta S_{\mathbf{k}^{\prime}}^{z} S_{\mathbf{q}^{\prime}}^{+}\right\rangle=\Delta\left(\mathbf{k}+\mathbf{k}^{\prime}\right) \Delta\left(\mathbf{q}-\mathbf{q}^{\prime}\right)\left\langle\delta S_{\mathbf{k}}^{z}(t) \delta S_{-\mathbf{k}}^{z}\right\rangle\left\langle S_{\mathbf{q}}^{-}(t) S_{\mathbf{q}}^{+}\right\rangle,
$$

где $\Delta(\mathbf{k})=1$ для векторов $\mathbf{k}=0, \mathbf{k}=\mathbf{G}$ обратной решетки и $\Delta(\mathbf{k})=0$ в остальных случаях. Рассмотрим приближенное вычисление корреляционных функций в (19).

Наиболее простой результат можно получить, если в уравнении (19) пренебречь зависимостью флуктуаций продольного спина от времени, полагая $\left\langle\delta S_{\mathbf{k}}^{z}(t) \delta S_{-\mathbf{k}}^{z}\right\rangle \simeq$ $\left\langle\delta S_{\mathbf{k}}^{z} \delta S_{-\mathbf{k}}^{z}\right\rangle=K^{z z}(\mathbf{k})$ при условии, что энергия этих возбуждений много меньше энергии поперечных возбуждений. При этом временну́ю корреляционную функцию поперечных флуктуаций спина можно вычислить в низшем приближении:

$$
\left\langle S_{\mathbf{q}}^{-}(t) S_{\mathbf{q}}^{+}\right\rangle=\int_{-\infty}^{\infty} d \omega e^{i \omega t} N(\omega)\left(-\frac{1}{\pi} \operatorname{Im} G^{(0)}(\mathbf{q}, \omega+i \delta)\right)=\sigma N\left(E_{\mathbf{q}}\right) e^{i E_{\mathbf{q}} t} .
$$

Здесь $N(\omega)=\left\{e^{\beta \omega}-1\right\}^{-1}$ и принято $h=0$. В этом приближении для массового оператора получаем формулу [17]

$$
M(\mathbf{q}, \omega)=\frac{1}{N} \sum_{\mathbf{k}} K^{z z}(\mathbf{k}) \frac{\left(J_{\mathbf{k}}-J_{\mathbf{q}-\mathbf{k}}\right)^{2}}{\omega-E_{\mathbf{q}-\mathbf{k}}} .
$$

Это выражение соответствует результатам вычисления массового оператора в диаграммной технике за счет рассеяния спиновых волн на продольных флуктуациях спина (см. формулу (12) в работе [7]).

Массовый оператор, обусловленный рассеянием спиновых возбуждений на поперечных компонентах спина, описывается многочастичной функцией (18), в которой для оператора продольной компоненты спина $\delta S_{i}^{z}$ используется соотношение $S_{i}^{z}=1 / 2-S_{i}^{-} S_{i}^{+}$. В этом представлении получим

$$
\left\langle Z_{j}^{-(\mathrm{irr})}(t) \mid Z_{i}^{+(\mathrm{irr})}\right\rangle^{(\mathrm{pp})}=\sum_{l, m} J_{j m} J_{l i} I_{j m, l i}(t),
$$


где

$$
I_{j m, l i}(t)=\left\langle\left[S_{m}^{-}(t) S_{m}^{+}(t) S_{j}^{-}(t)-S_{j}^{-}(t) S_{j}^{+}(t) S_{m}^{-}(t)\right]\left[S_{l}^{-} S_{l}^{+} S_{i}^{+}-S_{i}^{-} S_{i}^{+} S_{l}^{+}\right]\right\rangle .
$$

Приближение взаимодействующих мод для двухвременной корреляционной функции (21) определяется соотношением

$$
\begin{aligned}
I_{j m, l i}(t)= & {\left[\left\langle S_{m}^{+}(t) S_{l}^{-}\right\rangle-\left\langle S_{m}^{+}(t) S_{i}^{-}\right\rangle-\left\langle S_{j}^{+}(t) S_{l}^{-}\right\rangle+\left\langle S_{j}^{+}(t) S_{i}^{-}\right\rangle\right] \times } \\
& \times\left[\left\langle S_{m}^{-}(t) S_{l}^{+}\right\rangle\left\langle S_{j}^{-}(t) S_{i}^{+}\right\rangle+\left\langle S_{m}^{-}(t) S_{i}^{+}\right\rangle\left\langle S_{j}^{-}(t) S_{l}^{+}\right\rangle\right],
\end{aligned}
$$

где мы учли, что $j \neq m$ и $i \neq l$. Вводя фурье-разложение для корреляционных функций,

$$
\left\langle S_{i}^{-}(t) S_{j}^{+}\right\rangle=\frac{1}{N} \sum_{\mathbf{q}} e^{i \mathbf{q}(\mathbf{i}-\mathbf{j})} K^{-+}(\mathbf{q}, t),
$$

для неприводимой корреляционной функции в массовом операторе (17) получим представление

$$
\begin{aligned}
& \left\langle Z_{\mathbf{q}}^{-(\mathrm{irr})}(t) \mid Z_{\mathbf{q}}^{+(\mathrm{irr})}\right\rangle^{(\mathrm{pp})}=\frac{1}{N} \sum_{i, j} e^{i \mathbf{q}(\mathbf{i}-\mathbf{j})} \sum_{l, m} J_{j m} J_{l i} I_{j m, l i}(t)= \\
& =\frac{1}{2 N^{2}} \sum_{\mathbf{q}_{\mathbf{1}}, \mathbf{q}_{2}, \mathbf{q}_{3}}\left[V\left(\mathbf{q}_{1}, \mathbf{q}_{2}, \mathbf{q}_{3}\right)\right]^{2} K^{+-}\left(\mathbf{q}_{1}, t\right) K^{-+}\left(\mathbf{q}_{2}, t\right) K^{-+}\left(\mathbf{q}_{3}, t\right),
\end{aligned}
$$

где $\mathbf{q}_{\mathbf{1}}+\mathbf{q}_{\mathbf{2}}+\mathbf{q}_{3}=\mathbf{q}$. Вершина взаимодействия при учете симметрии по перестановке импульсов $\mathbf{q}_{2} \leftrightarrow \mathbf{q}_{3}$ в (23) определяется формулой

$$
\begin{aligned}
V\left(\mathbf{q}_{1}, \mathbf{q}_{2}, \mathbf{q}_{3}\right) & =J_{\mathbf{q}_{1}+\mathbf{q}_{2}}-J_{\mathbf{q}_{2}}+J_{\mathbf{q}_{1}+\mathbf{q}_{3}}-J_{\mathbf{q}_{3}}= \\
& =V_{\mathbf{k q p}}=J_{\mathbf{q}}-J_{\mathbf{q}-\mathbf{k}}+J_{\mathbf{k}-\mathbf{p}-\mathbf{q}}-J_{\mathbf{p}+\mathbf{q}}
\end{aligned}
$$

где мы ввели вершину $V_{\mathbf{k q p}}$ - эффективный потенциал взаимодействия спиновых волн в теории Дайсона [5], обычно используемый для описания четырехспинового взаимодействия (см., например, дополнение в книге [2]). При этом была проведена замена исходных переменных $\mathbf{q}_{1}=-\mathbf{p}, \mathbf{q}_{2}=\mathbf{p}+\mathbf{q}, \mathbf{q}_{3}=\mathbf{k}-\mathbf{q}$, где импульс при рассеянии удовлетворяет закону сохранения $\mathbf{q}_{1}+\mathbf{q}_{2}+\mathbf{q}_{3}=\mathbf{k}$.

Вводя фурье-компоненты временнь́х корреляционных функций и используя спектральные представления (20), для массового оператора после интегрирования по $d t^{\prime}$ и $d \omega^{\prime}$ в (17) получим выражение

$$
\begin{aligned}
M(\mathbf{q}, \omega)= & \frac{\sigma^{2}}{2 N^{2}} \sum_{\mathbf{q}_{1}, \mathbf{q}_{2}, \mathbf{q}_{3}} \Delta\left(\mathbf{q}_{1}+\mathbf{q}_{2}+\mathbf{q}_{3}-\mathbf{q}\right)\left[V\left(\mathbf{q}_{1}, \mathbf{q}_{2}, \mathbf{q}_{3}\right)\right]^{2} \times \\
& \times \iiint_{-\infty}^{+\infty} \frac{d \omega_{1} d \omega_{2} d \omega_{3}}{\omega_{2}+\omega_{3}-\omega_{1}-\omega} A\left(\mathbf{q}_{1}, \omega_{1}\right) A\left(\mathbf{q}_{2}, \omega_{2}\right) A\left(\mathbf{q}_{3}, \omega_{3}\right) \times \\
& \times\left\{N\left(\omega_{1}\right)\left[1+N\left(\omega_{2}\right)\right]\left[1+N\left(\omega_{3}\right)\right]-\left[1+N\left(\omega_{1}\right)\right] N\left(\omega_{2}\right) N\left(\omega_{3}\right)\right\},
\end{aligned}
$$

где использована спектральная функция спиновых возбуждений $A(\mathbf{q}, \omega)(16)$. Применяя нулевую ФГ (8) при вычислении спектральной функции $A^{(0)}(\mathbf{q}, \omega)=\delta\left(\omega-E_{\mathbf{q}}\right)$, 
для массового оператора (24) в низшем порядке по взаимодействию получим

$$
\begin{aligned}
M(\mathbf{q}, \omega)= & \frac{\sigma^{2}}{2 N^{2}} \sum_{\mathbf{q}_{1}, \mathbf{q}_{2}, \mathbf{q}_{3}} \Delta\left(\mathbf{q}_{1}+\mathbf{q}_{2}+\mathbf{q}_{3}-\mathbf{q}\right)\left[V\left(\mathbf{q}_{1}, \mathbf{q}_{2}, \mathbf{q}_{3}\right)\right]^{2} \times \\
& \times \frac{N\left(E_{\mathbf{q}_{1}}\right)\left[1+N\left(E_{\mathbf{q}_{2}}\right)+N\left(E_{\mathbf{q}_{3}}\right)\right]-N\left(E_{\mathbf{q}_{2}}\right) N\left(E_{\mathbf{q}_{3}}\right)}{E_{\mathbf{q}_{2}}+E_{\mathbf{q}_{3}}-E_{\mathbf{q}_{1}}-\omega},
\end{aligned}
$$

где мы учли соотношение

$$
\begin{aligned}
N\left(\omega_{1}\right)\left[1+N\left(\omega_{2}\right)\right][1 & \left.+N\left(\omega_{3}\right)\right]-\left[1+N\left(\omega_{1}\right)\right] N\left(\omega_{2}\right) N\left(\omega_{3}\right)= \\
& =N\left(\omega_{1}\right)\left[1+N\left(\omega_{2}\right)+N\left(\omega_{3}\right)\right]-N\left(\omega_{2}\right) N\left(\omega_{3}\right) .
\end{aligned}
$$

Выражение (25) для массового оператора соответствует результатам диаграммной техники (см. формулу (28б) в работе [7]) и приближению второго порядка в теории возмущений для временны́х ФГ (см. формулы (2.10) и (2.11) в работе [15]).

2.3. Низкотемпературная намагниченность. Для вычисления намагниченности можно воспользоваться точным соотношением для спиновых операторов $S=1 / 2$ :

$$
\sigma=2\left\langle S_{i}^{z}\right\rangle=1-2\left\langle S_{i}^{-} S_{i}^{+}\right\rangle=1-\frac{2}{N} \sum_{\mathbf{q}}\left\langle S_{\mathbf{q}}^{-} S_{\mathbf{q}}^{+}\right\rangle .
$$

Среднее значение корреляционной функции определяется формулой

$$
\left\langle S_{\mathbf{q}}^{-} S_{\mathbf{q}}^{+}\right\rangle=\sigma \int_{-\infty}^{\infty} d \omega N(\omega) A(\mathbf{q}, \omega)
$$

где использована спектральная функция (16). В обобщенном ПСП (9) получаем $\left\langle S_{\mathbf{q}}^{-} S_{\mathbf{q}}^{+}\right\rangle=\sigma N\left(h+E_{\mathbf{q}}\right)$, и уравнение для намагниченности принимает простой вид:

$$
\frac{1}{\sigma}=1+\frac{2}{N} \sum_{\mathbf{q}} N\left(h+E_{\mathbf{q}}\right) \equiv 1+2 P(h, T) .
$$

Эта формула была использована Тябликовым [2] для построения самосогласованной теории намагниченности в широкой области температур, включая парамагнитную фазу при $T>T_{\mathrm{C}}, h \neq 0$, где $T_{\mathrm{C}}$ - температура Кюри. При этом спектр спиновых волн вычислялся в ПСП, $E_{\mathbf{q}}^{(0)}=(\sigma / 2)\left(J_{0}-J_{\mathbf{q}}\right)$, без учета вкладов от статических флуктуаций спина в (10). В области низких температур $T \ll J_{0}$ среднее число спиновых возбуждений мало, $N\left(h+E_{\mathbf{q}}\right) \ll 1$, и для намагниченности можно написать разложение

$$
\sigma=\{1+2 P(h, T)\}^{-1}=1-2 P(h, T)+[2 P(h, T)]^{2}+\cdots .
$$

В ПСП получаем [2]

$$
P^{(0)}(h, T)=\frac{1}{N} \sum_{\mathbf{q}} N\left(h+E_{\mathbf{q}}^{(0)}\right)=Z_{3 / 2}\left(\frac{\tilde{h}}{\tau}\right) \tau^{3 / 2}+\frac{3 \pi}{4} Z_{5 / 2}\left(\frac{\tilde{h}}{\tau}\right) \tau^{5 / 2}+\cdots,
$$

где

$$
Z_{p}(x)=\sum_{n=1}^{\infty} n^{-p} e^{-n x}, \quad Z_{p}(0)=\zeta(p)
$$


При вычислении суммы по волновым векторам использован спектр для решетки со взаимодействием $z$ ближайших соседей, $J_{\mathbf{q}}=J_{0} z \gamma(\mathbf{q}), \gamma(\mathbf{q})=(1 / z) \sum_{\mathbf{a}} e^{i(\vec{q} \vec{a})}$, и введены безразмерные температура $\tau=3 T /\left(4 \pi z J_{0}\right)$ и магнитное поле $\tilde{h}=3 h /\left(4 \pi z J_{0}\right)$ (см. формулы (15.14)-(15.21) в работе [2]). В то время как первый член в (28) дает формулу Блоха для намагниченности $\left.\sigma(T)\right|_{T \rightarrow 0}=1-B T^{3 / 2}$, второй член $\propto[P(h, T)]^{2}$ приводит к поправкам $\propto T^{3}$, обусловленным кинематическим взаимодействием. Если использовать при вычислении суммы по волновым векторам в (29) спектр $E_{\mathbf{q}}(10)$ с учетом вкладов от флуктуаций спина, то мы получим вклады более высокого порядка по $T$ в функцию $P(h, T)$ в $(28)$, которые, однако, не компенсируют кинематический вклад $[P(h, T)]^{2}$, и вклад порядка $T^{3}$ в намагниченности сохранится (см., тем не менее, более общее представление спектра в теории первого порядка в работе [14]). Более того, если для вычисления низкотемпературной намагниченности в спиновой диаграммной технике использовать точное соотношение (26) и полученную в этой теории формулу для корреляционной функции поперечных компонент спина $\left\langle S_{i}^{-} S_{i}^{+}\right\rangle \propto \sigma$ (см. работу [7]), то такие же поправки $\propto P^{2} \propto T^{3}$ с необходимостью появятся в формуле (28) (в диаграммной технике для вычисления намагниченности используется теория возмущений для свободной энергии [6]). Таким образом, появление членов порядка $T^{3}$ в низкотемпературной намагниченности совершенно не связано с "расцеплением Тябликова", а является результатом применения теории низшего порядка, без учета вкладов от массового оператора в спектральной плотности (16) при вычислении корреляционной функции в (27). Более подробно эта проблема обсуждается в дополнении к монографии [2].

Покажем, что учет массового оператора (25) при вычислении корреляционной функции (27) приводит к компенсации кинематического вклада и исключению членов порядка $T^{3}$ в низкотемпературной намагниченности [18]. Чтобы приближенно учесть члены второго порядка по взаимодействию, запишем спектральную плотность (16) для $h=0$ в виде

$$
A(\mathbf{q}, \omega)=\frac{1}{\pi} \frac{\Gamma_{\mathbf{q}}(\omega)}{\left[\omega-E_{\mathbf{q}}-M^{\prime}(\mathbf{q}, \omega)\right]^{2}+\Gamma_{\mathbf{q}}^{2}(\omega)} \simeq\left(1-\alpha_{\mathbf{q}}\right) \delta\left(\omega-\widetilde{E}_{\mathbf{q}}\right)+\frac{1}{\pi} \frac{\Gamma_{\mathbf{q}}(\omega)}{\left(\omega-\widetilde{E}_{\mathbf{q}}\right)^{2}},
$$

где введена перенормированная энергия спиновых возбуждений $\widetilde{E}_{\mathbf{q}}=E_{\mathbf{q}}+M^{\prime}\left(\mathbf{q}, E_{\mathbf{q}}\right)$, а также их затухание $\Gamma_{\mathbf{q}}(\omega)=-M^{\prime \prime}(\mathbf{q}, \omega)$. Весовой множитель $\alpha_{\mathbf{q}}$, определяющий квазичастичный вклад, находится из правила сумм:

$$
\int_{-\infty}^{\infty} d \omega A(\mathbf{q}, \omega)=1, \quad \alpha_{\mathbf{q}}=\frac{1}{\pi} \int_{-\infty}^{\infty} d \omega \frac{\Gamma_{\mathbf{q}}(\omega)}{\left(\omega-\widetilde{E}_{\mathbf{q}}\right)^{2}}
$$

Учитывая выражение для массового оператора (25), находим

$$
\begin{aligned}
\Gamma_{\mathbf{q}}(\omega)= & \frac{\pi \sigma^{2}}{2 N^{2}} \sum_{\mathbf{q}_{1}, \mathbf{q}_{2}, \mathbf{q}_{3}}\left[V\left(\mathbf{q}_{1}, \mathbf{q}_{2}, \mathbf{q}_{3}\right)\right]^{2} \delta\left(E_{\mathbf{q}_{2}}+E_{\mathbf{q}_{3}}-E_{\mathbf{q}_{1}}-\omega\right) \times \\
& \times\left\{N\left(E_{\mathbf{q}_{1}}\right)\left[1+N\left(E_{\mathbf{q}_{2}}\right)+N\left(E_{\left.\mathbf{q}_{3}\right)}\right]-N\left(E_{\mathbf{q}_{2}}\right) N\left(E_{\mathbf{q}_{3}}\right)\right\} .\right.
\end{aligned}
$$

В этом приближении получим корреляционную функцию (27):

$$
\left\langle S_{\mathbf{q}}^{-} S_{\mathbf{q}}^{+}\right\rangle=\sigma N\left(\widetilde{E}_{\mathbf{q}}\right)+\frac{\sigma}{\pi} \int_{-\infty}^{\infty} d \omega \frac{\left[N(\omega)-N\left(\widetilde{E}_{\mathbf{q}}\right)\right] \Gamma_{\mathbf{q}}(\omega)}{\left(\omega-\widetilde{E}_{\mathbf{q}}\right)^{2}} .
$$


Соответственно, спиновую корреляционную функцию запишем в виде

$$
\begin{aligned}
\left\langle S_{i}^{-} S_{i}^{+}\right\rangle & =\frac{\sigma}{N} \sum_{\mathbf{q}} N\left(\widetilde{E}_{\mathbf{q}}\right)+\frac{\sigma}{N} \sum_{\mathbf{q}} \frac{1}{\pi} \int_{-\infty}^{\infty} d \omega \frac{\left[N(\omega)-N\left(\widetilde{E}_{\mathbf{q}}\right)\right] \Gamma_{\mathbf{q}}(\omega)}{\left(\omega-\widetilde{E}_{\mathbf{q}}\right)^{2}} \equiv \\
& \equiv \sigma\left[P^{(1)}(T)+P^{(2)}(T)\right],
\end{aligned}
$$

где

$$
\begin{aligned}
P^{(2)}(T)= & \frac{\sigma^{2}}{2 N^{3}} \sum_{\mathbf{q}, \mathbf{q}_{1}, \mathbf{q}_{2}, \mathbf{q}_{3}}\left[V\left(\mathbf{q}_{1}, \mathbf{q}_{2}, \mathbf{q}_{3}\right)\right]^{2}\left[N\left(E_{\mathbf{q}_{2}}+E_{\mathbf{q}_{3}}-E_{\mathbf{q}_{1}}\right)-N\left(\widetilde{E}_{\mathbf{q}}\right)\right] \times \\
& \times \frac{N\left(E_{\mathbf{q}_{1}}\right)\left[1+N\left(E_{\mathbf{q}_{2}}\right)+N\left(E_{\mathbf{q}_{3}}\right)\right]-N\left(E_{\mathbf{q}_{2}}\right) N\left(E_{\mathbf{q}_{3}}\right)}{\left(E_{\mathbf{q}_{2}}+E_{\mathbf{q}_{3}}-E_{\mathbf{q}_{1}}-E_{\mathbf{q}}\right)^{2}} .
\end{aligned}
$$

В низкотемпературном пределе поправки второго порядка оцениваются величиной $P^{(2)} \simeq 2\left[P^{(0)}\right]^{2}\left(1+2 P^{(0)}\right)($ см. формулы $(2.27),(2.32)$ в работе [15]). В результате в уравнении для намагниченности $(28)$

$$
\sigma=[1+2 P(T)]^{-1} \simeq 1-2\left\{P^{(0)}(T)+2\left[P^{(0)}(T)\right]^{2}\right\}+\left[2 P^{(0)}(T)\right]^{2} \simeq 1-2 P^{(0)}(T)
$$

происходит компенсация кинематического вклада $\left[2 P^{(0)}(T)\right]^{2} \propto T^{3}$ за счет динамического взаимодействия-рассеяния спиновых волн (30), как и в теории Дайсона [5]. Для получения более строгих результатов необходимо рассмотреть приближение $T$-матрицы рассеяния при вычислении массового оператора (см. работу [15]).

\section{3. ПАРАМАГНИТНАЯ СПИНОВАЯ ВОСПРИИМЧИВОСТЬ}

В этом разделе мы рассмотрим модель Гейзенберга (1) со взаимодействием только ближайших соседей, $J_{\mathbf{i}, \mathbf{i}+\mathbf{a}}=J$, и вычислим динамическую спиновую восприимчивость (2) в парамагнитной фазе.

3.1. Метод функции релаксации. В парамагнитной фазе с нулевой намагниченностью среднее значение коммутатора $\left\langle\left[S_{i}^{+}, S_{j}^{-}\right]\right\rangle=2 \delta_{i j}\left\langle S_{i}^{z}\right\rangle$ равно нулю. Поскольку эта величина входит в качестве неоднородного члена в уравнение (4) для ФГ, удобнее рассматривать уравнение движения для функции релаксации Кубо-Мори [20], [27]:

$$
((A \mid B))_{\omega}=-i \int_{0}^{\infty} d t e^{i \omega t}(A(t), B),
$$

где $(A(t), B)$ - скалярное произведение Кубо-Мори, определяемое выражением

$$
(A(t), B)=\int_{0}^{\beta} d \lambda\langle A(t-i \lambda) B\rangle .
$$

Функция релаксации (31) связана с ФГ (3) уравнением

$$
\omega((A \mid B))_{\omega}=\langle\langle A \mid B\rangle\rangle_{\omega}-\langle\langle A \mid B\rangle\rangle_{\omega=0} .
$$

Для этих функций можно получить также соотношения

$$
\begin{aligned}
((i \dot{A} \mid B))_{\omega} & =((A \mid-i \dot{B}))_{\omega}=\langle\langle A \mid B\rangle\rangle_{\omega}, \\
(i \dot{A}, B) & =(A,-i \dot{B})=\langle[A, B]\rangle, \\
(A, B) & =-\langle\langle A \mid B\rangle\rangle_{\omega=0} .
\end{aligned}
$$


Согласно уравнению (33) динамическую спиновую восприимчивость (2) можно определить, вычисляя спиновую функцию релаксации $\Phi(\mathbf{q}, \omega)=\left(\left(S_{\mathbf{q}}^{+} \mid S_{-\mathbf{q}}^{-}\right)\right)_{\omega}$ :

$$
\chi(\mathbf{q}, \omega)=\chi_{\mathbf{q}}-\omega \Phi(\mathbf{q}, \omega),
$$

где $\chi_{\mathbf{q}} \equiv \chi(\mathbf{q}, 0)=\left(S_{\mathbf{q}}^{+}, S_{-\mathbf{q}}^{-}\right)$- статическая спиновая восприимчивость. Используя метод уравнения движения, получим следующую систему уравнений для функции релаксации:

$$
\omega\left(\left(S_{\mathbf{q}}^{+} \mid S_{-\mathbf{q}}^{-}\right)\right)_{\omega}=\chi_{\mathbf{q}}+\left(\left(i \dot{S}_{\mathbf{q}}^{+} \mid S_{-\mathbf{q}}^{-}\right)\right)_{\omega}, \quad \omega\left(\left(i \dot{S}_{\mathbf{q}}^{+} \mid S_{-\mathbf{q}}^{-}\right)\right)_{\omega}=\left(\left(i \dot{S}_{\mathbf{q}}^{+} \mid-i \dot{S}_{-\mathbf{q}}^{-}\right)\right)_{\omega}
$$

где в последнем уравнении согласно (35) неоднородный член в парамагнитной фазе равен нулю, $\left(i \dot{S}_{\mathbf{q}}^{+}, S_{-\mathbf{q}}^{-}\right)=\left\langle\left[S_{\mathbf{q}}^{+}, S_{-\mathbf{q}}^{-}\right]\right\rangle=(2 / \sqrt{N})\left\langle S_{\mathbf{q}=0}^{z}\right\rangle=0$.

Воспользуемся далее методом проектирования Мори аналогично рассмотренному выше для $\Phi Г$. Для этого запишем уравнение для операторов спинового тока $i \dot{S}_{\mathbf{q}}^{ \pm}$в виде

$$
i \dot{S}_{\mathbf{q}}^{ \pm}=\left[S_{\mathbf{q}}^{ \pm}, H\right]=\omega_{\mathbf{q}}^{(0)} S_{\mathbf{q}}^{ \pm}+\left\{i \dot{S}_{\mathbf{q}}^{ \pm}\right\}^{(\mathrm{irr})}, \quad\left(\left\{i \dot{S}_{\mathbf{q}}^{ \pm}\right\}^{(\mathrm{irr})}, S_{-\mathbf{q}}^{\mp}\right)=0,
$$

где неприводимые части операторов определяются из условия ортогональности в терминах скалярного произведения Кубо-Мори (32). При этом линейный член в уравнении (39), в отличие от уравнения (6) для $Ф Г$, в парамагнитной фазе вклада не дает:

$$
\omega_{\mathbf{q}}^{(0)}=\frac{\left(i \dot{S}_{\mathbf{q}}^{ \pm}, S_{-\mathbf{q}}^{\mp}\right)}{\left(S_{\mathbf{q}}^{ \pm}, S_{-\mathbf{q}}^{\mp}\right)}=\frac{\left\langle\left[S_{\mathbf{q}}^{ \pm}, S_{-\mathbf{q}}^{\mp}\right]\right\rangle}{\chi_{\mathbf{q}}}=0 .
$$

Следовательно, неприводимые части операторов (39) равны исходным операторам: $\left\{i \dot{S}_{\mathbf{q}}^{ \pm}\right\}^{(\mathrm{irr})}=i \dot{S}_{\mathbf{q}}^{ \pm}$. В результате решения системы уравнений (38) для функции релаксации мы можем написать уравнение

$$
\Phi(\mathbf{q}, \omega)=\Phi^{(0)}(\mathbf{q}, \omega)+\Phi^{(0)}(\mathbf{q}, \omega) T(\mathbf{q}, \omega) \Phi^{(0)}(\mathbf{q}, \omega), \quad \Phi^{(0)}(\mathbf{q}, \omega)=\frac{\chi_{\mathbf{q}}}{\omega},
$$

где матрица рассеяния первого порядка определена как функция релаксации токов:

$$
T(\mathbf{q}, \omega)=\frac{1}{\chi_{\mathbf{q}}^{2}}\left(\left(i \dot{S}_{\mathbf{q}}^{+} \mid-i \dot{S}_{-\mathbf{q}}^{-}\right)\right)_{\omega}
$$

Чтобы учесть только спроектированную временну́ю зависимость корреляционных функций в матрице рассеяния (41), как и в методе ФГ, мы определяем собственную часть матрицы, которая не содержит частей, соединенных одной функцией релаксации нулевого порядка $\Phi^{(0)}(\mathbf{q}, \omega)$. Собственная часть определяет функцию памяти $M(\mathbf{q}, \omega)$ согласно уравнению

$$
T(\mathbf{q}, \omega)=\frac{M(\mathbf{q}, \omega)}{\chi_{\mathbf{q}}^{2}}+\frac{M(\mathbf{q}, \omega)}{\chi_{\mathbf{q}}^{2}} \Phi^{(0)}(\mathbf{q}, \omega) T(\mathbf{q}, \omega) .
$$

В терминах функции памяти решение уравнения (40) записывается в виде

$$
\Phi(\mathbf{q}, \omega)=\left(\left(S_{\mathbf{q}}^{+} \mid S_{-\mathbf{q}}^{-}\right)\right)_{\omega}=\frac{\chi_{\mathbf{q}}}{\omega-M(\mathbf{q}, \omega) / \chi_{\mathbf{q}}},
$$


где функция памяти выражается через операторы спинового тока $i \dot{S}_{\mathbf{q}}^{ \pm}$:

$$
M(\mathbf{q}, \omega)=\chi_{\mathbf{q}}^{2} T_{\mathbf{q}}^{(\mathrm{pp})}(\omega)=\left(\left(i \dot{S}_{\mathbf{q}}^{+} \mid-i \dot{S}_{-\mathbf{q}}^{-}\right)\right)_{\omega}^{(\mathrm{pp})} .
$$

В отличие от ферромагнитного состояния, где операторы $S_{\mathbf{q}}^{ \pm}$соответствуют операторам рождения и уничтожения квазичастиц, и поэтому уравнения первого порядка позволяют получить спектр квазичастиц в ПСП (10) и найти поправки за счет массового оператора (15) от операторов тока $J_{i}^{ \pm}=(i d / d t) S_{i}^{ \pm}$, в парамагнитном случае необходимо рассмотреть уравнения второго порядка для операторов спиновой плотности $S_{\mathbf{q}}^{ \pm}$, где спектр возбуждений определяется операторами силы $F_{i}^{ \pm}=i^{2}\left(d^{2} / d t^{2}\right) S_{i}^{ \pm}$. Для этого продифференцируем двухвременное представление функции памяти $\left(\left(i \dot{S}_{\mathbf{q}}^{+}(t) \mid-i \dot{S}_{-\mathbf{q}}^{-}\left(t^{\prime}\right)\right)\right.$ по двум временам $t$ и $t^{\prime}$. В результате получим следующие уравнения:

$$
\begin{aligned}
\omega\left(\left(i \dot{S}_{\mathbf{q}}^{+} \mid-i \dot{S}_{-\mathbf{q}}^{-}\right)\right)_{\omega} & =m(\mathbf{q})+\left(\left(-\ddot{S}_{\mathbf{q}}^{+} \mid-i \dot{S}_{-\mathbf{q}}^{-}\right)\right)_{\omega} \\
\omega\left(\left(-\ddot{S}_{\mathbf{q}}^{+} \mid-i \dot{S}_{-\mathbf{q}}^{-}\right)\right)_{\omega} & =\left(\left(-\ddot{S}_{\mathbf{q}}^{+} \mid-\ddot{S}_{-\mathbf{q}}^{-}\right)\right)_{\omega}
\end{aligned}
$$

где в (44) мы ввели статическую корреляционную функцию

$$
m(\mathbf{q})=\left(i \dot{S}_{\mathbf{q}}^{+} \mid-i \dot{S}_{-\mathbf{q}}^{-}\right)=\left\langle\left[i \dot{S}_{\mathbf{q}}^{+}, S_{-\mathbf{q}}^{-}\right]\right\rangle
$$

При этом неоднородный член в уравнении (45) равен нулю,

$$
\left(-\ddot{S}_{\mathbf{q}}^{+} \mid-i \dot{S}_{-\mathbf{q}}^{-}\right)=\left\langle\left[-\ddot{S}_{\mathbf{q}}^{+}, S_{-\mathbf{q}}^{-}\right]\right\rangle=\frac{1}{\pi} \int_{-\infty}^{+\infty} d \omega \omega^{2} \operatorname{Im} \chi_{\mathbf{q}}(\omega+i \delta)=0,
$$

поскольку все четные моменты мнимой части восприимчивости, являющейся нечетной функцией от частоты $\omega$, равны нулю. Далее мы повторим процедуру проецирования для операторов силы $F_{i}^{ \pm}=i^{2}\left(d^{2} / d t^{2}\right) S_{i}^{ \pm}$аналогично (39):

$$
-\ddot{S}_{\mathbf{q}}^{ \pm}=\left[i \dot{S}_{\mathbf{q}}^{ \pm}, H\right]=\omega_{\mathbf{q}}^{(1)} i \dot{S}_{\mathbf{q}}^{ \pm}+\left\{-\ddot{S}_{\mathbf{q}}^{ \pm}\right\}^{(\mathrm{irr})}, \quad\left(\left\{-\ddot{S}_{\mathbf{q}}^{ \pm}\right\}^{(\mathrm{irr})}, i \dot{S}_{-\mathbf{q}}^{\mp}\right)=0 .
$$

При этом, как показано выше, неоднородный член в уравнении (47) равен нулю: $\omega_{\mathbf{q}}^{(1)}=\left(-\ddot{S}_{\mathbf{q}}^{ \pm},-i \dot{S}_{-\mathbf{q}}^{\mp}\right) /\left(i \dot{S}_{\mathbf{q}}^{ \pm},-i \dot{S}_{-\mathbf{q}}^{\mp}\right)=0$. Следовательно, неприводимые части операторов (47) равны исходным операторам, $\left\{-\ddot{S}_{\mathbf{q}}^{ \pm}\right\}^{(\mathrm{irr})}=-\ddot{S}_{\mathbf{q}}^{ \pm}$.

По аналогии с выводом уравнений для функции релаксации (40), матрицы рассеяния (41) и функции памяти (42) мы можем записать решение системы уравнений (44), (45) для функции памяти в виде

$$
M(\mathbf{q}, \omega)=\frac{m(\mathbf{q})}{\omega-\Sigma(\mathbf{q}, \omega)}
$$

где массовый оператор представляет собой собственную часть матрицы рассеяния второго порядка:

$$
\Sigma(\mathbf{q}, \omega)=\frac{1}{m(\mathbf{q})}\left(\left(-\ddot{S}_{\mathbf{q}}^{+} \mid-\ddot{S}_{-\mathbf{q}}^{-}\right)\right)_{\omega}^{(\mathrm{pp})}
$$


Используя представления (43) для функции релаксации и (48) для функции памяти, получим окончательное выражение для функции релаксации через массовый оператор (49):

$$
\Phi(\mathbf{q}, \omega)=\chi_{\mathbf{q}} \frac{\omega-\Sigma(\mathbf{q}, \omega)}{\omega^{2}-\omega_{\mathbf{q}}^{2}-\omega \Sigma(\mathbf{q}, \omega)},
$$

где мы ввели частоту спиновых флуктуаций в статическом пределе:

$$
\omega_{\mathbf{q}}^{2}=\frac{m(\mathbf{q})}{\chi_{\mathbf{q}}}
$$

Следовательно, динамическая спиновая восприимчивость согласно (37) принимает вид

$$
\chi(\mathbf{q}, \omega)=\chi_{\mathbf{q}}-\omega \Phi(\mathbf{q}, \omega)=-\frac{m(\mathbf{q})}{\omega^{2}-\omega_{\mathbf{q}}^{2}-\omega \Sigma(\mathbf{q}, \omega)} .
$$

Полученное выражение для динамической спиновой восприимчивости с массовым оператором в виде (49) совпадает по форме с результатами работ [20], [30]. Спектр спиновых флуктуаций определяется спектральной плотностью (мнимой частью динамической спиновой восприимчивости (51)):

$$
B(\mathbf{q}, \omega)=\frac{1}{\pi} \chi^{\prime \prime}(\mathbf{q}, \omega)=\frac{1}{\pi} \frac{-\omega \Sigma^{\prime \prime}(\mathbf{q}, \omega) m(\mathbf{q})}{\left[\omega^{2}-\omega_{\mathbf{q}}^{2}-\omega \Sigma^{\prime}(\mathbf{q}, \omega)\right]^{2}+\left[\omega \Sigma^{\prime \prime}(\mathbf{q}, \omega)\right]^{2}} ;
$$

здесь $\Sigma\left(\mathbf{q}, \omega+i 0^{+}\right)=\Sigma^{\prime}(\mathbf{q}, \omega)+i \Sigma^{\prime \prime}(\mathbf{q}, \omega)$, где $\Sigma^{\prime}(\mathbf{q}, \omega)=-\Sigma^{\prime}(\mathbf{q},-\omega)$ и $\Sigma^{\prime \prime}(\mathbf{q}, \omega)=$ $\Sigma^{\prime \prime}(\mathbf{q},-\omega)$ - действительная и мнимая части массового оператора соответственно.

3.2. Статическая спиновая восприимчивость. В методе функции релаксации в отличие от метода ФГ для определения статической спиновой восприимчивости $\chi_{\mathbf{q}}$ необходимо независимо вычислить частоту спиновых флуктуаций $\omega_{\mathbf{q}}^{2}(50)$ в статическом пределе. В работе [20] эта функция была найдена из уравнений для функции релаксации в высокочастотном пределе, а в работе [30] для этого использовалось спектральное представление $\chi_{\mathbf{q}}=\int_{-\infty}^{\infty}(d \omega / \pi \omega) \chi^{\prime \prime}(\mathbf{q}, \omega)$. Для вычисления $\omega_{\mathbf{q}}^{2}$ аналогично работам [31], [32] мы воспользуемся более простым методом, основанным на приближенном решении уравнения

$$
\left\langle\left[i \dot{S}_{\mathbf{q}}^{+}, S_{-\mathbf{q}}^{-}\right]\right\rangle=\left(-\ddot{S}_{\mathbf{q}}^{+}, S_{-\mathbf{q}}^{-}\right) .
$$

Левую часть этого уравнения легко вычислить:

$$
m(\mathbf{q})=\left\langle\left[i \dot{S}_{\mathbf{q}}^{+}, S_{-\mathbf{q}}^{-}\right]\right\rangle=8 J\left(1-\gamma_{\mathbf{q}}\right) C_{10},
$$

где введена спиновая корреляционная функция для узлов $i, j\left(\mathbf{R}_{i j}=\mathbf{i}-\mathbf{j}\right)$

$$
C_{i j}=\left\langle S_{i}^{+}, S_{j}^{-}\right\rangle=\frac{1}{N} \sum_{\mathbf{q}} \cos \left(\mathbf{q} \mathbf{R}_{i j}\right) C_{\mathbf{q}}, \quad C_{\mathbf{q}}=\left\langle S_{\mathbf{q}}^{+} S_{-\mathbf{q}}^{-}\right\rangle .
$$


Вычислим корреляционную функцию в правой части (53), записывая ее в виде

$$
\left(-\ddot{S}_{\mathbf{q}}^{+}, S_{-\mathbf{q}}^{-}\right)=\sum_{\mathbf{R}_{i l}} e^{-i \mathbf{q} \mathbf{R}_{i l}}\left(-\ddot{S}_{i}^{+}, S_{l}^{-}\right),
$$

где $-\ddot{S}_{i}^{+}=\left[\left[S_{i}^{+}, H\right], H\right]$ определяется уравнением

$$
-\ddot{S}_{i}^{+}=h^{2} S_{i}^{+}+\sum_{j \neq n} J_{i j}\left\{J_{j n}\left(P_{j i n}-P_{n i j}\right)-J_{i n}\left(P_{i j n}-P_{n j i}\right)\right\},
$$

в котором $P_{j i n}=\left(S_{j}^{z} S_{i}^{z}+S_{j}^{-} S_{i}^{+} / 2\right) S_{n}^{+}$. Подставляя (56) в уравнение $(55)$, получим многоузельные корреляционные функции, которые мы вычислим в приближении взаимодействующих мод аналогично соотношению (22) для двухвременных корреляционных функций. При этом появляются уравнения следующего типа:

$$
\left(S_{i}^{+} S_{j}^{-} S_{n}^{+}, S_{l}^{-}\right) \simeq \alpha_{1}\left\langle S_{n}^{+} S_{j}^{-}\right\rangle\left(S_{i}^{+}, S_{l}^{-}\right)+\alpha_{2}\left\langle S_{i}^{+} S_{j}^{-}\right\rangle\left(S_{n}^{+}, S_{l}^{-}\right) .
$$

Здесь аналогично работам [31], [32] введены множители $\alpha_{1}$ и $\alpha_{2}$, учитывающие приближенный характер расцепления с помощью перенормировки вершин для спиновых корреляционных функций ближайших и следующих за ближайшими соседями соответственно. Определение этих множителей должно проводиться при численном расчете статической восприимчивости на основе правил сумм, например $C_{i, i}=\left\langle S_{i}^{+} S_{i}^{-}\right\rangle=(2 / 3) S(S+1)=1 / 2$.

В результате приближенного вычисления многочастичных корреляционных функций мы можем записать исходное уравнение (55) в виде

$$
\left(-\ddot{S}_{\mathbf{q}}^{+}, S_{-\mathbf{q}}^{-}\right) \simeq \omega_{\mathbf{q}}^{2}\left(S_{\mathbf{q}}^{+}, S_{-\mathbf{q}}^{-}\right)=\omega_{\mathbf{q}}^{2} \chi_{\mathbf{q}}
$$

где частота спиновых флуктуаций в ПСП определяется уравнением

$$
\omega_{\mathbf{q}}^{2}=2 J^{2}\left(1-\gamma_{\mathbf{q}}\right)\left[1-2 \alpha_{1} C_{01}\left(1+4 \gamma_{\mathbf{q}}\right)+2 \alpha_{2}\left(2 C_{11}+C_{02}\right)\right] .
$$

Следовательно, статическая восприимчивость согласно (50) имеет вид

$$
\chi_{\mathbf{q}}=\frac{m(\mathbf{q})}{\omega_{\mathbf{q}}^{2}}=\frac{4 C_{10}}{J\left[1-2 \alpha_{1} C_{01}\left(1+4 \gamma_{\mathbf{q}}\right)+2 \alpha_{2}\left(2 C_{11}+C_{02}\right)\right]} .
$$

Температура Кюри $T_{\mathrm{C}}$ перехода из парамагнитной фазы в упорядоченную ферромагнитную фазу определяется из условия $\chi_{\mathbf{q}} \rightarrow \infty$. Расходимость статической восприимчивости возникает за счет роста с понижением температуры корреляционной функции $C_{10}>0$ ближайших соседей. При этом знаменатель обращается в ноль для волнового вектора $\mathbf{q}=0$, для которого $\gamma_{\mathbf{q}}=1$, так что температура Кюри определяется уравнением $10 \alpha_{1} C_{10}=2 \alpha_{2}\left(2 C_{11}+C_{02}\right)$. Для антиферромагнитной модели Гейзенберга с обменным взаимодействием $J<0$ корреляционная функция ближайших соседей отрицательна, $C_{10}<0$. В результате расходимость статической восприимчивости возникает для антиферромагнитного волнового вектора $\mathbf{q}=\mathbf{Q}$, для которого $\gamma_{\mathbf{q}}=-1$, и температура Нееля $T_{\mathrm{N}}$ определяется уравнением $6 \alpha_{1} C_{10}=2 \alpha_{2}\left(2 C_{11}+C_{02}\right)$, где $C_{11}>0$ и $C_{02}>0$ (см. работу [23]). 
3.3. Массовый оператор. Выразим массовый оператор (49) через неприводимую часть временно́й корреляционной функции сил аналогично формуле (17):

$$
\begin{aligned}
\Sigma(\mathbf{q}, \omega)= & \frac{1}{m(\mathbf{q})}\left(\left(-\ddot{S}_{\mathbf{q}}^{+} \mid-\ddot{S}_{-\mathbf{q}}^{-}\right)\right)_{\omega}^{(\mathrm{pp})}=\frac{1}{2 \pi m(\mathbf{q})} \int_{-\infty}^{\infty} d \omega^{\prime} \frac{e^{\beta \omega^{\prime}}-1}{\omega^{\prime}\left(\omega-\omega^{\prime}\right)} \times \\
& \times \frac{1}{N} \sum_{i, j} e^{-i \mathbf{q}(\mathbf{i}-\mathbf{j})} \int_{-\infty}^{\infty} d t e^{-i \omega^{\prime} t}\left\langle F_{i}^{-}(t) \mid F_{j}^{+}\right\rangle^{(\mathrm{pp})}
\end{aligned}
$$

где оператор силы $F_{i}^{ \pm}=-\ddot{S}_{i}^{ \pm}$в координатном представлении определяется уравнением (56). Для вычисления неприводимой части временно́й корреляционной функции в правой части соотношения (58) воспользуемся приближением взаимодействующих мод, как и в уравнении $(22)$ в методе ФГ:

$$
\left\langle S_{i}^{z}(t) S_{j}^{z}(t) S_{n}^{-}(t) S_{k}^{z} S_{l}^{z} S_{m}^{+}\right\rangle=\left\langle S_{n}^{-}(t) S_{m}^{+}\right\rangle\left[\left\langle S_{i}^{z}(t) S_{k}^{z}\right\rangle\left\langle S_{j}^{z}(t) S_{l}^{z}\right\rangle+\left\langle S_{i}^{z}(t) S_{l}^{z}\right\rangle\left\langle S_{j}^{z}(t) S_{k}^{z}\right\rangle\right] .
$$

Используя спектральные представления для временных корреляционных функций в последнем соотношении и интегрируя по $d t^{\prime}$ и $d \omega^{\prime}$ аналогично (24), для мнимой части массового оператора (58) получим выражение

$$
\begin{aligned}
\Sigma^{\prime \prime}(\mathbf{q}, \omega)= & \frac{\pi(2 J)^{4}\left(e^{\beta \omega}-1\right)}{2 m(\mathbf{q}) \omega} \frac{1}{N^{2}} \sum_{\mathbf{q}_{1}, \mathbf{q}_{2}} W\left(\mathbf{q}_{1}, \mathbf{q}_{2}, \mathbf{q}_{3}\right) \iiint_{-\infty}^{\infty} d \omega_{1} d \omega_{2} d \omega_{3} \times \\
& \times N\left(\omega_{1}\right) N\left(\omega_{2}\right) N\left(\omega_{3}\right) \delta\left(\omega_{1}+\omega_{2}+\omega_{3}-\omega\right) A_{\mathbf{q}_{1}}\left(\omega_{1}\right) A_{\mathbf{q}_{2}}\left(\omega_{2}\right) A_{\mathbf{q}_{3}}\left(\omega_{3}\right),
\end{aligned}
$$

где $\mathbf{q}_{3}=\mathbf{q}-\mathbf{q}_{1}-\mathbf{q}_{2}$. Спектральная плотность $A_{\mathbf{q}}(\omega)$ определяется формулой (52). Вершина взаимодействия спиновых возбуждений имеет вид

$$
W\left(\mathbf{q}_{1}, \mathbf{q}_{2}, \mathbf{q}_{3}\right)=\left\{\Gamma_{\mathbf{q}_{1} \mathbf{q}_{2} \mathbf{q}_{3}}^{2}+\Gamma_{\mathbf{q}_{1} \mathbf{q}_{2} \mathbf{q}_{3}} \Gamma_{\mathbf{q}_{2} \mathbf{q}_{1} \mathbf{q}_{3}}\right\},
$$

где

$$
\Gamma_{\mathbf{q}_{1} \mathbf{q}_{2} \mathbf{q}_{3}}=4\left(\gamma_{\mathbf{q}_{3}+\mathbf{q}_{1}}-\gamma_{\mathbf{q}_{2}}\right)\left(\gamma_{\mathbf{q}_{3}}-\gamma_{\mathbf{q}_{1}}\right)-\gamma_{\mathbf{q}_{1}}+\gamma_{\mathbf{q}_{3}}+\gamma_{\mathbf{q}_{2}+\mathbf{q}_{3}}-\gamma_{\mathbf{q}_{2}+\mathbf{q}_{1}} .
$$

При выводе формулы (59) использовалось соотношение $\left\langle\left\langle S_{\mathbf{q}}^{z} \mid S_{-\mathbf{q}}^{z}\right\rangle_{\omega}=\left\langle\left\langle S_{\mathbf{q}}^{+} \mid S_{-\mathbf{q}}^{-}\right\rangle\right\rangle_{\omega} / 2\right.$, имеющее место в парамагнитной фазе. Подставляя массовый оператор в общее выражение для динамической спиновой восприимчивости (51) и учитывая выражение (57) для статической частоты $\omega_{\mathbf{q}}$, мы можем определить спектр спиновых возбуждений с учетом неупругих процессов их рассеяния.

При этом возможно провести самосогласованное вычисление спектра, пользуясь методом итераций. Вычисляя массовый оператор (59) со спектральными интенсивностями в ПСП, $A_{\mathbf{q}}^{(0)}(\omega)=\left[m(\mathbf{q}) / 2 \omega_{\mathbf{q}}\right]\left[\delta\left(\omega-\omega_{\mathbf{q}}\right)-\delta\left(\omega+\omega_{\mathbf{q}}\right)\right]$, находим выражение для массового оператора и спектральной интенсивности (52) в низшем порядке по взаимодействию. Полученное выражение для спектральной интенсивности можно использовать при вычислении массового оператора (59) в приближениях следующего порядка. В общем случае требуется проведение достаточно сложных численных расчетов. Подобные расчеты были проведены для определения спектра спиновых возбуждений в рамках более общей $t-J$ модели с антиферромагнитным обменом $(J<0)$, как в нормальной фазе [23], так и в сверхпроводящей фазе [24].

Можно, однако, сделать некоторые общие замечания о характере спектра спиновых возбуждений в пределах низких и высоких частот. Рассмотрим выражение для 
спиновой восприимчивости в пределе $\omega \rightarrow 0$ низких частот. Действительная часть массового оператора является нечетной функцией от частоты, и поэтому ее вкладом $\operatorname{Re} \Sigma(\mathbf{q}, \omega) \propto \omega$ можно пренебречь, а мнимая часть является четной функцией и имеет конечный предел. В этом случае асимптотическое выражение для $\chi(\mathbf{q}, \omega)$ может быть записано в виде

$$
\chi(\mathbf{q}, \omega)=\frac{m(\mathbf{q})}{\omega_{\mathbf{q}}^{2}-i \omega \Gamma(\mathbf{q})}=\chi_{\mathbf{q}} \frac{i \gamma(\mathbf{q})}{\omega+i \gamma(\mathbf{q})},
$$

где $\Gamma(\mathbf{q})=-\lim _{\omega \rightarrow 0} \operatorname{Im} \Sigma\left(\mathbf{q}, \omega+i 0^{+}\right)-$затухание, $\gamma(\mathbf{q})=\omega_{\mathbf{q}}^{2} / \Gamma(\mathbf{q})$. Следовательно, в низкочастотном пределе следует ожидать, что спиновые флуктуации имеют релаксационное поведение с характерным затуханием $\gamma(\mathbf{q})$.

В области высоких частот, где определяемое мнимой частью массового оператора затухание может быть мало по сравнению с частотой спиновых флуктуаций, асимптотическое выражение для спиновой восприимчивости можно представить в виде

$$
\chi(\mathbf{q}, \omega)=\frac{m(\mathbf{q})}{\omega_{\mathbf{q}}^{2}+\Omega_{\mathbf{q}}^{2}-\omega^{2}},
$$

где высокочастотная перенормировка частоты определяется выражением $\Omega_{\mathbf{q}}^{2}=$ $\lim _{\omega \rightarrow \infty} \omega \operatorname{Re} \Sigma(\mathbf{q}, \omega)$. В этом случае спиновые возбуждения могут носить характер распространяющихся квазичастиц. Общий анализ спектра возбуждений в рамках анизотропной модели Гейзенберга был проведен в работе [20].

\section{4. ОБСУЖДЕНИЕ}

Представленный в настоящей работе метод вычисления двухвременных ФГ [1] на основе дифференцирования ФГ по двум временам и применения проекционной техники типа Мори имеет ряд преимуществ по сравнению с методом “расцепления" ФГ при последовательном дифференцировании ФГ по одному времени [3]. Метод проектирования исключает произвол в методе расцеплений $Ф Г$, а дифференцирование по двум временам позволяет получить точное уравнение типа Дайсона с массовым оператором в виде многочастичной ФГ. Во многих случаях вычисление массового оператора в приближении взаимодействующих мод оказывается вполне достаточным для получения разумных физических результатов, соответствующих вычислению массового оператора в приближении непересекающихся диаграмм в диаграммной технике. При этом точность того или иного приближения в методе уравнений движения для двухвременных ФГ нетрудно проконтролировать, сопоставляя его с соответствующими диаграммами в температурной диаграммной технике. Определенным недостатком этого метода является невозможность обратной процедуры определения расцепления, которое соответствовало бы выбранному ряду диаграмм.

Однако простота построения цепочки уравнений в случае операторов со сложными коммутационными соотношениями, таких как спиновые операторы или операторы Хаббарда, диаграммная техника для которых необычайно сложна [10], в значительной степени компенсирует недостатки метода уравнений движения для двухвременных ФГ. В качестве примера подобных вычислений для сложных моделей можно привести работы по изучению модели Хаббарда [33], сверхпроводимости в $t-J$ модели [34] и в модели Хаббарда [35] при использовании техники операторов 
Хаббарда. Этот метод оказался необычайно удобным при анализе спектра фононов в сильно ангармонических кристаллах при учете ангармонического взаимодействия всех порядков [36]. С помощью метода уравнений движения для ФГ удается сформулировать теорию самосогласованных фононов, спектр которых зависит от корреляционных функций смещений атомов в решетке; данные функции вычисляются с помощью этого же спектра. В результате аналогично теории Тябликова ренормированных спиновых волн получается самосогласованная система уравнений, что позволяет исследовать спектр фононов вплоть до температуры неустойчивости решетки, определяющей верхнюю границу температуры плавления кристалла [37].

Благодарности. Автор благодарен Ю. Г. Рудому за полезные обсуждения.

\section{Список литературы}

[1] Н. Н. Боголюбов, С. В. Тябликов, Докл. АН СССР, 126:1 (1959), 53-56.

[2] С. В. Тябликов, Методъ квантовой теории магнетизма, ред. Д. Н. Зубарев, Ю. Г. Рудой, Наука, М., 1975.

[3] Д. Н. Зубарев, УФН, 71:5 (1960), 71-116; Д. Н. Зубарев, В. Г. Морозов, Г. Рёпке, Статистическая механика неравновесных прочессов, Физматлит, М., 2002.

[4] С. В. Тябликов, Укр. матем. журн., 11 (1959), 287-294.

[5] F. Dyson, Phys. Rev., 102:5 (1956), 1217-1230.

[6] В. Г. Вакс, А. И. Ларкин, С. А. Пикин, ЖЭТФ, 53:1 (1967), 281-299.

[7] В. Г. Вакс, А. И. Ларкин, С. А. Пикин, ЖЖЭТФ, 53:3 (1967), 1089-1106.

[8] Ю. А. Изюмов, Ф. А. Кассан-Оглы, ФММ, 30:2 (1970), 225-234.

[9] В. Г. Барьяхтар, В.Н.Криворучко, Д. А. Яблонский, ЖЭТФ, 85 (1983), 601-613.

[10] Ю. А. Изюмов, Ф. А. Кассан-Оглы, Ю. Н. Скрябин, Полевые методы в теории ферромагнетизма, Наука, М., 1974.

[11] Ю.А. Изюмов, Ю.Н. Скрябин, Статистическая механика магнитоупорядоченных систем, Наука, М., 1987.

[12] В. Г. Барьяхтар, В.Н.Криворучко, Д. А. Яблонский, Функиии Грина в теории магнетизма, Наукова думка, Киев, 1984.

[13] Ю. А. Церковников, ТМФ, 7:2 (1971), 250-261.

[14] Ю. Г. Рудой, Ю. А. Церковников, ТМФ, 14:1 (1973), 102-122.

[15] Ю. Г. Рудой, Ю. А. Церковников, ТМФ, 15:3 (1973), 388-406.

[16] H. Mori, Progr. Theor. Phys., 33:3 (1965), 423-455; 34:3, 399-416.

[17] N. M. Plakida, Phys. Lett. A., 43:6 (1973), 481-482.

[18] Н. М. Плакида, П. Р. Русек, Никзотемпературная намагниченность гейзенберговского ферромагнетика, Препринт ОИЯИ Р4-8032, ОИЯИ, Дубна, 1974.

[19] Ю. А. Церковников, ТМФ, 49:2 (1981), 219-233.

[20] Ю. А. Церковников, ТМФ, 52:1 (1982), 147-160.

[21] Ю. А. Церковников, ТМФ, 154:1 (2008), 197-206.

[22] А. А. Владимиров, Д. Иле, Н. М. Плакида, ТМФ, 145:2 (2005), 240-255.

[23] A. A. Vladimirov, D. Ihle, N. M. Plakida, Phys. Rev. B, 80:10 (2009), 104425, 12 pp.

[24] A. A. Vladimirov, D. Ihle, N. M. Plakida, Phys. Rev. B, 83 (2011), 024411.

[25] Yu. A. Izyumov, B. M. Letfulov, J. Phys.: Condens. Matter, 3:28 (1991), 5373-5391.

[26] N.M. Plakida, High-Temperature Cuprate Superconductors. Experiment, Theory, and Applications, Springer Series in Solid-State Sciences, 166, Springer, Heidelberg, Berlin, 2010.

[27] Д. Форстер, Гидродинамические флуктуации, нарушенная симметрия и корреляционные функции, Атомиздат, М., 1980. 
[28] В. П. Калашников, ТМФ, 34:3 (1978), 412-425.

[29] M. Ichiyanagi, J. Phys. Soc. Jpn., 32:3 (1972), 604-609.

[30] I. Sega, P. Prelošek, J. Bonča, Phys. Rev. B, 68:5 (2003), 054524, 6 pp.

[31] H. Shimahara, S. Takada, J. Phys. Soc. Jpn., 60:7 (1991), 2394-2405.

[32] S. Winterfeldt, D. Ihle, Phys. Rev. B, 59:9 (1999), 6010-6012.

[33] F. Mancini, A. Avella, Advances in Physics, 53:5-6 (2004), 537-768.

[34] N. M. Plakida, V.S. Oudovenko, Phys. Rev. B, 59:18 (1999), 11949-11961.

[35] Н. М. Плакида, Л. Антон, С. Адам, Г. Адам, ЖЭЭФ, 124:2 (2003), 367-378.

[36] Н. М. Плакида, "Метод двухвременных функций Грина в теории ангармонических кристаллов", Статистическая физика и квантовая теория поля, ред. Н. Н. Боголюбов, Наука, М., 1973, 205-240.

[37] Н. М. Плакида, ФТT, 11:3 (1969), 700-707.

Поступила в редакцию 24.02.2011 